\title{
Erratum to: Tumor suppressor miR-145 reverses drug resistance by directly targeting DNA damage-related gene RAD18 in colorectal cancer
}

\author{
Ye Dong ${ }^{1} \cdot$ Rui-Lei Liu ${ }^{2}$ - Yan-Zhen Deng ${ }^{1} \cdot$ Wen-Jun Wang ${ }^{3} \cdot$ Wei-Dong Li $^{1}$
}

Published online: 18 February 2016

(C) International Society of Oncology and BioMarkers (ISOBM) 2016

Erratum to: Tumor Biol. (2015) 36:5011-5019

DOI 10.1007/s13277-015-3152-5

The author group, affiliation and article note sections for this published online article unfortunately contained mistakes.

The corrected author group is shown above and its corresponding affiliation is shown below.

In the article note section, the former article note should be corrected as Ye Dong and Rui-lei Liu contributed equally to this paper.

Ye Dong and Rui-lei Liu contributed equally to this paper.

The online version of the original article can be found at http://dx.doi.org/ 10.1007/s13277-015-3152-5.

Wei-Dong Li

weidongli2012@gmail.com

1 Department of the First Medicine, Cancer Hospital of Guangzhou, Medical College, Guangzhou 510095, China

2 Department of Thyroid \& Breast Surgery, The Third Affiliated Hospital of Sun Yet-Sen University, Guangzhou 510630, China

3 State Key Laboratory of Respiratory Diseases, Guangzhou Institute of Respiratory Disease, The First Affiliated Hospital of Guangzhou Medical University, Guangzhou 510120, China 